\title{
Axial Symmetry Cosmological Constant Vacuum Solution of Field Equations with a Curvature Singularity, Closed Time-Like Curves, and Deviation of Geodesics
}

\author{
Faizuddin Ahmed $\mathbb{D}^{1}{ }^{1}$ Bidyut Bikash Hazarika, ${ }^{2}$ and Debojit Sarma ${ }^{2}$ \\ ${ }^{1}$ Maryam Ajmal Women's College of Science \& Technology, Hojai, 782435 Assam, India \\ ${ }^{2}$ Department of Physics, Cotton University, Guwahati, 781001 Assam, India \\ Correspondence should be addressed to Faizuddin Ahmed; faizuddinahmed15@gmail.com
}

Received 28 May 2020; Revised 19 August 2020; Accepted 3 November 2020; Published 19 November 2020

Academic Editor: Diego Saez Chillon Gomez

Copyright (@) 2020 Faizuddin Ahmed et al. This is an open access article distributed under the Creative Commons Attribution License, which permits unrestricted use, distribution, and reproduction in any medium, provided the original work is properly cited. The publication of this article was funded by $\mathrm{SCOAP}^{3}$.

In this paper, we present a type $\mathrm{D}$, nonvanishing cosmological constant, vacuum solution of Einstein's field equations, extension of an axially symmetric, asymptotically flat vacuum metric with a curvature singularity. The space-time admits closed time-like curves (CTCs) that appear after a certain instant of time from an initial space-like hypersurface, indicating it represents a time-machine space-time. We wish to discuss the physical properties and show that this solution can be interpreted as gravitational waves of Coulomb-type propagate on anti-de Sitter space backgrounds. Our treatment focuses on the analysis of the equation of geodesic deviations.

\section{Introduction}

Closed time-like curves constitute one of the most intriguing aspects of general relativity. The first solution of the field equations admitting closed time-like curves (CTCs) is the Gödel rotating Universe [1]. It represents a rotating universe and is axially symmetric, given by

$d s^{2}=d r^{2}+d z^{2}+\left(\sinh ^{2} r-\sinh ^{4} r\right) d \theta^{2}+2 \sqrt{2} \sinh ^{2} r d \theta d t-d t^{2}$

The coordinates are in the ranges $0 \leq r<\infty,-\infty<z<\infty$, and $-\infty<t<\infty$, and $\theta$ is periodic. For some $r>r_{0}$, the metric function, $g_{\theta \theta}=\sinh ^{2} r-\sinh ^{4} r$, becomes negative. The circle defined by $r>r_{0}$ and $t=0=z$ will be time-like everywhere. This condition is fulfilled when $r>r_{0}=\ln (1+\sqrt{2})$ which is the condition for the existence of CTCs in the Gödel space-time because one of the coordinates $\theta \in[0,2 \pi]$ is periodic. The next one is the van Stockum space-time [2], which predates the Gödel solution and was shown later to have CTCs [3]. Examples of space-time admitting CTCs including
NUT-Taub metric [4-7], Kerr and Kerr-Newman black hole solution [8-10], Gott time-machine [11], Grant space-time [12], Krasnikov tube [13], Bonnor's metrics [14-19], and others [20-36]. Space-time with causality violating curves is classified as either eternal or true time-machine spacetimes. In eternal time machine space-time case, CTCs always preexist. In this category would be [1] or [2] (see also, Refs. $[23,25,27,32,36])$. A true time machine space-time is the one in which CTCs evolve at a particular instant of time from an initial space-like hypersurface in a causally well-behaved manner satisfying all the energy conditions with known type of matter fields. In this category, the Ori time machine spacetime [37] is considered to be most remarkable. But the matter sources satisfying all the energy conditions are of unknown type in this space-time. Most of the time machine models suffer from one or more drawbacks. For space-time admitting CTCs, the matter-energy sources must be realistic, that is, the stress-energy tensor must be of a known type of matter fields, which satisfy all the energy conditions. Many spacetime model, for examples, traversable wormholes $[38,39]$ and warp drive models [40-43] violate the weak energy condition (WEC), which states that $T_{\mu \nu} U^{\mu} U^{\nu} \geq 0$ for a time-like 
tangent vector field $U^{\mu}$, that is, the energy-density must be nonnegative. Some other space-times admitting CTCs violate the strong energy condition (SEC) (e.g., Refs. [44-47]), which states that $\left(T_{\mu \nu}-1 / 2 g_{\mu \nu} T\right) U^{\mu} U^{\nu} \geq 0$. In addition, some solutions do not admit a partial Cauchy surface (initial space-like hypersurface) (e.g., Refs. [1, 48]) and/or CTCs come from infinity (e.g., Refs. $[11,12])$. In addition, there is a curvature singularity in some solutions admitting CTCs [3, 35, 48-53].

In literature, only a handful of solutions of Einstein's field equations with the stress-energy tensor in $[1,33,34]$ and type $\mathrm{N}$ Einstein space-time in [30] have a negative cosmological constant. In this work, we try to construct a type D Einstein space-time with a negative cosmological constant which was not studied earlier. The cosmological constant plays a vital role in explaining the dynamics of the universe. A tiny positive cosmological constant neatly explains the late-time accelerated expansion of the universe. Indeed, our universe is observed to be undergoing a de Sitter (dS) type expansion in the present epoch. For a negative cosmological constant, space-time is labelled as an anti-de Sitter (AdS) space. The AdS space has been a subject of intense study in recent times on account of the celebrated AdS/CFT correspondence [54], which provides a link between a quantum theory of gravity on an asymptotically AdS space and a lower-dimensional conformal field theory (CFT) on its boundary.

\section{Review of a Type D Vacuum Space-Time with a Curvature Singularity and CTCs [51]}

In Ref. [51], a type D axially symmetric, asymptotically flat vacuum solution of the field equations with zero cosmological constant, was constructed. This vacuum metric is as follow

$$
\begin{aligned}
d s^{2}= & -\cosh t \operatorname{coth} t \sinh ^{2} r d t^{2}+\cosh ^{2} r \sinh r d r^{2} \\
& +\operatorname{csch} r d z^{2}+\sinh ^{2} r\left(2 \sqrt{2} \cosh t d t d \phi-\sinh t d \phi^{2}\right)
\end{aligned}
$$

After doing a number of transformations into the above metric, we arrive at the following

$$
d s^{2}=\cosh ^{2} r \sinh r d r^{2}+\operatorname{csch} r d z^{2}-\sinh ^{2} r\left(2 d t d \phi+t d \phi^{2}\right)
$$

The Kretschmann scalar of the above metric is

$$
K=R_{\mu \nu \rho \sigma} R^{\mu \nu \rho \sigma}=\frac{12}{\sinh ^{6} r} .
$$

For constant $r, z$, the metric (3) reduces to conformal Misner metric in 2D

$$
d s^{2}=\Omega\left(-2 d t d \phi-t d \phi^{2}\right)
$$

where $\Omega=\sinh ^{2} r$ is the conformal factor.
In the context of CTCs, the Misner space metric in 2D is interesting because CTCs appear after a certain instant of time from causally well-behaved conditions. The metric for the Misner space in 2D [55] is given by

$$
d s_{\mathrm{Mis}}^{2}=-2 d T d X-T d X^{2}
$$

where $-\infty<T<\infty$ but the coordinate $X$ is periodic locally. The metric (6) is regular everywhere as det $g=-1$ including at $T=0$. The curves $T=T_{0}$, where $T_{0}$ is a constant, are closed since $X$ is periodic. The curves $T<0$ are spacelike, $T>0$ are time-like, while the null curves $T=0$ form the chronology horizon. The second type of curves, namely, $T=T_{0}>0$, are closed time-like curves. Therefore, the metric (2) or (3) is a four-dimensional generalization of Misner space in curved space-time. Note that the above space-time is the vacuum solution of field equations, a Ricci flat, that is, $R_{\mu \nu}=0$. Li [56] constructed a Misner-like AdS space-time, a timemachine model. Levanony and Ori [57] constructed a three-, four-dimensional generalization of flat Misner space metric.

In this paper, we extend the above Ricci flat space-time (2) to the Einstein space-times of Petrov type D, which satisfy the following conditions

$$
R_{\mu \nu}=\Lambda g_{\mu \nu}, R=4 \Lambda, \Lambda<0 \text { or } \Lambda>0
$$

It is an anti-de Sitter-like space if $\Lambda<0$ and de Sitter like if $\Lambda>0$. The extended space-time satisfies all the basic requirements (see details in Ref. [30]) for a time machine space-time except one, that is, this new model is not free from curvature singularity.

\section{Analysis of a Cosmological Constant Vacuum Space-Time}

Consider the following line element, a modification of the metric (2) given by

$$
\begin{aligned}
d s^{2}= & \sinh ^{2} r\left(-\cosh t \operatorname{coth} t d t^{2}+2 \sqrt{2} \cosh t d t d \phi-\sinh t d \phi^{2}\right) \\
& +\frac{d r^{2}}{\left(\alpha \operatorname{csch} r \operatorname{sech}^{2} r+\beta^{2} \tanh ^{2} r\right)}+\left(\alpha \operatorname{csch} r+\beta^{2} \sinh ^{2} r\right) d z^{2}
\end{aligned}
$$

Here, $\alpha$ is a positive constant, and $\beta$ is real. The coordinates are labelled $x^{0}=t, x^{1}=r, x^{2}=\phi$, and $x^{3}=z$. The ranges of the coordinates are

$$
-\infty<t<\infty, 0 \leq r<\infty,-\infty<z<\infty,
$$

and $\phi$ is a periodic coordinate $\phi \sim \phi+\phi_{0}$, with $\phi_{0}>0$. The metric is Lorentzian with signature $(-,+,+,+)$ and the determinant of the corresponding metric tensor $g_{\mu v}$,

$$
\operatorname{det} g=-\cosh ^{2} r \sinh ^{4} r \cosh ^{2} t
$$


Now, we have evaluated the Ricci tensor $R_{\mu \nu}$ of the spacetime (8) as follows:

$$
\begin{aligned}
& R_{t t}=3 \beta^{2} \cosh t \operatorname{coth} t \sinh ^{2} r, \\
& R_{t \phi}=R_{\phi t}=-3 \sqrt{2} \beta^{2} \cosh t \sinh ^{2} r, \\
& R_{r r}=-3 \beta^{2}\left(\frac{\cosh ^{2} r}{\alpha \operatorname{csch} r+\beta^{2} \sinh ^{2} r}\right), \\
& R_{\phi \phi}=3 \beta^{2} \sinh ^{2} r \sinh t, \\
& R_{z z}=-3 \beta^{2}\left(\alpha \operatorname{csch} r+\beta^{2} \sinh ^{2} r\right) .
\end{aligned}
$$

The Ricci scalar is given by

$$
R_{\mu}^{\mu}=R=-12 \beta^{2}
$$

Using the metric tensor components of the above spacetime, we have found that the Ricci tensor

$$
R_{\mu \nu}=-3 \beta^{2} g_{\mu \nu}(\mu, v=0,1,2,3),
$$

and the Einstein tensor $G_{\mu \nu}$ are

$$
G_{v}^{\mu}=3 \beta^{2} \operatorname{diag}(1,1,1,1) .
$$

From the Einstein's field equations $G_{\mu \nu}+\Lambda g_{\mu \nu}=0$ and from eq. (14), we have

$$
3 \beta^{2}=-\Lambda \Rightarrow \beta= \pm \sqrt{-\frac{\Lambda}{3}}, \Lambda<0 .
$$

Thus, from the above analysis, it is clear that the spacetime considered by (8) is an example of the class of Einstein space of anti-de Sitter-type and satisfies eq. (7) for a negative cosmological constant. We have shown later that the spacetime possesses a curvature singularity at $r \rightarrow 0$.

An interesting property of the metrics (8) is that it reduces to 2D Misner space metric [55] for constant $r, z$. For that, we do the following transformations

$$
t \rightarrow \sinh ^{-1} t, \phi \rightarrow \phi+(\sqrt{2}+1) \ln t
$$

into the metric (8) (replacing $\beta^{2} \rightarrow-\Lambda / 3$ ), we arrive at the following line element

$$
d s^{2}=\sinh ^{2} r\left(-2 d t d \phi-t d \phi^{2}\right)+\frac{\cosh ^{2} r d r^{2}}{\left(\alpha \operatorname{csch} r-(\Lambda / 3) \sinh ^{2} r\right)}
$$

$$
+\left(\alpha \operatorname{csch} r-\frac{\Lambda}{3} \sinh ^{2} r\right) d z^{2}
$$

For constant $r=r_{0}>0$ and $z=z_{0}$, the metric (17) becomes

$$
d s_{\mathrm{conf}}^{2}=\sinh ^{2} r\left(-2 d t d \phi-t d \phi^{2}\right)=\Omega d s_{\mathrm{Mis}}^{2}
$$

a conformal Misner space metric in $2 \mathrm{D}$ where $\Omega$ is the conformal factor. Therefore, the space-time admits CTC for $t=t_{0}$ $>0$ similar to the Misner space discussed earlier.

We check whether the CTCs evolve from an initially space-like $t=$ constant hypersurface (and thus $t$ is a time coordinate). This is determined by calculating the norm of the vector $\nabla_{\mu} t$ [37] (or alternately from the value of $g^{t t}$ in the inverse metric tensor $\left.g^{\mu v}\right)$. A hypersurface $t=$ constant is space-like when $g^{t t}<0$ at $t<0$, time-like when $g^{t t}>0$ for $t>0$, and null $g^{t t}=0$ for $t=0$. For the metric (8), we have

$$
\nabla_{\mu} t \nabla^{\mu} t=g^{t t}=\frac{\sinh t}{\sinh ^{2} r \cosh ^{2} t} .
$$

Thus, a hypersurface $t=$ constant is spacelike for $t<0$, time-like for $t>0$, and null at $t=0$. We restrict our analysis to $r>0$; otherwise, no CTCs will be formed. Thus, the space-like $t=$ constant $<0$ hypersurface can be chosen as initial hypersurface over which initial data may be specified. There is a Cauchy horizon at $t=t_{0}=0$ called the chronology horizon, which separates the causal past and future in a pastdirected and future-directed manner. Hence, the space-time evolves from a partial Cauchy surface (i.e., initial space-like hypersurface) in a causally well-behaved, up to a moment, i.e., a null hypersurface $t=t_{0}=0$ and the formation of CTCs takes place from causally well-behaved initial conditions. The evolution of CTC is thus identical to the case of the Misner space.

That the space-time represented by (8) satisfies the requirements of axial symmetry is clear from the following. Consider the Killing vector $\eta=\partial_{\phi}$ having the normal form

$$
\eta^{\mu}=(0,0,1,0)
$$

Its covector form

$$
\eta_{\mu}=\sinh ^{2} r(\sqrt{2} \cosh t, 0,-\sinh t, 0)
$$

The vector (22) satisfies the Killing equation $\eta_{\mu ; \nu}+$ $\eta_{v ; \mu}=0$. The space-time is axial symmetry if the norm of the Killing vector $\eta^{\mu}$ vanishes on the axis i.e., at $r=0$ (see $[58,59]$ and references therein). In our case

$$
X=\left|\eta_{\mu} \eta^{\mu}\right|=\left|g_{\phi \phi}\right|=\left|-\sinh t \sinh ^{2} r\right| \rightarrow 0,
$$

as $r \rightarrow 0$.

The metric has a curvature singularity at $r=0$. We find that the Kretschmann scalar is

$$
K=R_{\mu \nu \rho \sigma} R^{\mu \nu \rho \sigma}=\frac{8 \Lambda^{2}}{3}+\frac{12 \alpha^{2}}{\sinh ^{6} r} .
$$


We can see that the scalar curvature diverges at $r \rightarrow 0$, which indicates that the space-time possesses a curvature singularity. In addition, the Kretschmann scalar becomes $K \rightarrow$ $8 \Lambda^{2} / 3$ for $r \rightarrow \infty$, indicating that the metric (8) is asymptotically anti-de Sitter-like space radially [60].

3.1. Classification and Physical Interpretation of the SpaceTimes. Here, we first classify the space-time according to the Petrov classification scheme and then analyze the effect of local fields of the solution. We construct a set of null tetrad $(k, l, m, \bar{m})[61]$ for the space-time (8). Explicitly, these covectors are

$$
\begin{aligned}
& k_{\mu}=\frac{\sinh r}{\sqrt{2}}\left(\frac{\cosh t}{\sqrt{\sinh t}}, 0,(-\sqrt{2}+1) \sqrt{\sinh t}, 0\right) \text {, } \\
& l_{\mu}=\frac{\sinh r}{\sqrt{2}}\left(\frac{\cosh t}{\sqrt{\sinh t}}, 0,-(\sqrt{2}+1) \sqrt{\sinh t}, 0\right) \\
& m_{\mu}=\frac{1}{\sqrt{2}}\left(0, \frac{\cosh r}{\sqrt{\alpha \operatorname{csch} r-(\Lambda / 3) \sinh ^{2} r}}, 0, i \sqrt{\alpha \operatorname{csch} r-\frac{\Lambda}{3} \sinh ^{2} r}\right) \text {, }
\end{aligned}
$$

$\bar{m}_{\mu}=\frac{1}{\sqrt{2}}\left(0, \frac{\cosh r}{\sqrt{\alpha \operatorname{csch} r-(\Lambda / 3) \sinh ^{2} r}}, 0,-i \sqrt{\alpha \operatorname{csch} r-\frac{\Lambda}{3} \sinh ^{2} r}\right)$.

The set of null tetrad above is such that the metric tensor for the line element (8) can be expressed as

$$
g_{\mu \nu}=-k_{\mu} l_{v}-l_{\mu} k_{v}+m_{\mu} \bar{m}_{v}+\bar{m}_{\mu} m_{v}
$$

The vectors (25), (26), (27), and (28) are null vector and orthogonal, except for $k_{\mu} l^{\mu}=-1$ and $m_{\mu} \bar{m}^{\mu}=1$.

We calculate the five Weyl scalars, of these only

$$
\Psi_{2}=C_{\mu \nu \rho \sigma} k^{\mu} m^{v} \bar{m}^{\rho} l^{\sigma}=\frac{\alpha}{2 \sinh ^{3} r},
$$

is nonvanishing, while the rest are vanish. Thus, the metric is clearly of type D in the Petrov classification scheme.

We set up an orthonormal frame $e_{(a)}=\left\{e_{(0)}, e_{(1)}, e_{(2)}\right.$, $\left.e_{(3)}\right\}, e_{(a)} \cdot e_{(b)} \equiv e_{(a)}^{\mu} e_{(b)}^{v} g_{\mu \nu}=\eta_{a b}=\operatorname{diag}(-1,+1,+1,+1)$, which consists of three space-like unit vectors $e_{(i)}, i=1,2,3$ and one time-like vector $e_{(0)}[62]$. Notations are such that small Latin indices are raised and lowered with Minkowski metric $\eta^{a b}$, $\eta_{a b}$, and Greek indices are raised and lowered with metric tensor $g^{\mu \nu}, g_{\mu \nu}$. The dual basis is $e^{(i)}=e_{(i)}$ and $e^{(0)}=-e_{(0)}$. These frame components in terms of tetrad vector can be expressed as

$k=\frac{1}{\sqrt{2}}\left(e_{(0)}+e_{(2)}\right), l=\frac{1}{\sqrt{2}}\left(e_{(0)}-e_{(2)}\right), m=\frac{1}{\sqrt{2}}\left(e_{(1)}+i e_{(3)}\right)$.
In order to analyze the effect of local gravitational fields of these solutions, we have used the equations of geodesic deviation $[25,33,52,63-66]$ which in terms of orthonormal frame $e_{(a)}$ are

$$
\ddot{Z}^{(i)}=-R_{(0)(j)(0)}^{(i)} Z^{(j)}, i, j=1,2,3,
$$

where $e_{(0)}=u$ is a time-like four-velocity vector of the free test particles. We set here $Z^{(0)}=0$ such that all test particles are synchronized by the proper time. From the standard definition of the Weyl tensor and the Einstein's field equation for zero the stress-energy tensor, we get (see Eq. (4) in [66])

$$
R_{(i)(0)(j)(0)}=C_{(i)(0)(j)(0)}-\frac{\Lambda}{3} \delta_{i j},
$$

where $C_{(i)(0)(j)(0)} \equiv e_{(i)}^{\mu} u^{v} e_{(j)}^{\rho} u^{\sigma} C_{\mu \nu \rho \sigma}$ are the components of the Weyl tensor.

The only nonvanishing Weyl scalars are given by (30) so that

$$
C_{(1)(0)(1)(0)}=-\Psi_{2}=C_{(3)(0)(3)(0)}, C_{(2)(0)(2)(0)}=2 \Psi_{2}
$$

Therefore, the equations of geodesic deviation (32) take the following form

$$
\begin{aligned}
\ddot{Z}^{(1)} & =-R_{(0)(j)(0)}^{(1)} Z^{(j)}=-\left(C_{(1)(0)(1)(0)}-\frac{\Lambda}{3}\right) Z^{(1)} \\
& =\left(\Psi_{2}+\frac{\Lambda}{3}\right) Z^{(1)},
\end{aligned}
$$

$$
\begin{aligned}
\ddot{Z}^{(2)} & =-R_{(0)(j)(0)}^{(2)} Z^{(j)}=-\left(C_{(2)(0)(2)(0)}-\frac{\Lambda}{3}\right) Z^{(2)} \\
& =\left(-2 \Psi_{2}+\frac{\Lambda}{3}\right) Z^{(2)} \\
\ddot{Z}^{(3)} & =-R_{(0)(j)(0)}^{(3)} Z^{(j)}=-\left(C_{(3)(0)(3)(0)}-\frac{\Lambda}{3}\right) Z^{(3)} \\
& =\left(\Psi_{2}+\frac{\Lambda}{3}\right) Z^{(3)} .
\end{aligned}
$$

In the limit $\alpha \rightarrow 0$, all the Weyl scalars including $\Psi_{2}$ vanishes. In this limit, the space-time (8) becomes anti-de Sitter (AdS) space. So the equations of geodesic deviation (35) in this limit reduces to

$$
\ddot{Z}^{(i)}=\frac{\Lambda}{3} Z^{(i)}
$$


with the solutions

$$
\begin{aligned}
& Z^{(i)}=a_{i} \tau+b_{i} \text { for } \Lambda=0 \\
& Z^{(i)}=a_{i} \cos \left(\sqrt{-\frac{\Lambda}{3} \tau}\right)+b_{i} \sin \left(\sqrt{-\frac{\Lambda}{3} \tau}\right) \text { for } \Lambda<0,
\end{aligned}
$$

where $a_{i}, b_{i}, i=1,2,3$ are the arbitrary constants.

Again, in the limit $\Lambda \rightarrow 0$, that is, $\beta \rightarrow 0$, the only nonvanishing Weyl scalars is $\Psi_{2}$ given by (30). The space-time (8) reduces to type D vacuum space-time of zero cosmological constant with a curvature singularity which we discussed, in detail in Ref. [51]. In this limit $(\Lambda \rightarrow 0)$, the equations of geodesic deviation (35) becomes

$$
\ddot{Z}^{(1)}=\Psi_{2} Z^{(1)}, \ddot{Z}^{(2)}=-2 \Psi_{2} Z^{(2)}, \ddot{Z}^{(3)}=\Psi_{2} Z^{(3)},
$$

with the solutions

$$
\begin{aligned}
& Z^{(1)}=c_{1} \cosh \left(\sqrt{\Psi_{2}} \tau\right)+d_{1} \sinh \left(\sqrt{\Psi_{2}} \tau\right), \\
& Z^{(2)}=c_{2} \cos \left(\sqrt{2 \Psi_{2}} \tau\right)+d_{2} \sin \left(\sqrt{2 \Psi_{2}} \tau\right), \\
& Z^{(3)}=c_{3} \cosh \left(\sqrt{\Psi_{2}} \tau\right)+d_{3} \sinh \left(\sqrt{\Psi_{2}} \tau\right),
\end{aligned}
$$

where $c_{i}, d_{i}, i=1,2,3$ are the arbitrary constants and $\Psi_{2} \neq 0$.

\section{Summary and Future Work}

In this paper, we generalize a Ricci flat space-time [51] to the case of nonvanishing cosmological constant solution in fourdimensional curved space-time, still represent vacuum solutions of the Einstein's field equations are the generalization of 2D Misner space metric. By introducing a cosmological constant term into the metric components in the metric [51], we have seen that for $r=r_{0}$, and $z=z_{0}$, where $r_{0}, z_{0}$ are constants, these space-times reduce to $2 \mathrm{D}$ conformal Misner space geometry. As discussed in Section 2, the Misner space metric admits CTCs which appear after a certain instant of time from causally well-behaved conditions. Thus, the presented metrics as well as the one studied in [51] evolve CTC from an initial space-like hypersurface at a certain instant of time. Though causality violating space-times have been studied extensively in the literature, few of them belongs to true time-machine space-time (e.g., [24-26, 28, 31, 33, 34, 37]), and others in (e.g., [26, 51-53]) are lacking one or more basic requirements for a true time-machine space-time. In addition, many time-machine models mentioned in the introduction violate one or more the energy condition. Our space-time is the vacuum solution of Einstein's field equations of nonzero cosmological constant. So all the energy conditions are automatically satisfied, and the modified metrics would represent true time-machine space-time but lacking the property of being free from curvature singularity. Furthermore, we have analyzed the space-time and discussed their physical properties. It was demonstrated that these space-times can be understood as gravitational waves of Coulomb-types which propagate on anti-de Sitter backgrounds. A positive cosmological constant $(\Lambda)$ plays an important role in explaining the dynamics of the universe. But in our case, however, it is negative. One can use this modified space-time as a model to study the quantum gravity in connection to the quantum field theory. The dynamic stability of the modified space-times is beyond the scope of this work. Our motivation to further study this problem is to construct a space-time metric which satisfies all criteria for a true time-machine, like obeying the energy conditions, realistic or known types of matter sources, singularity-free and evolves CTCs from an initial space-like hypersurface in a causally well-behaved manner after a certain instant of time.

\section{Data Availability}

There is no data associated with this manuscript or no data have been used to prepare it.

\section{Conflicts of Interest}

The authors declare that there is no conflict of interest regarding publication of this paper.

\section{Acknowledgments}

The authors sincerely acknowledge the anonymous kind referees for their valuable comments and constructive suggestions.

\section{References}

[1] K. Godel, "An example of a new type of cosmological solutions of Einstein's field equations of gravitation," Reviews of Modern Physics, vol. 21, no. 3, pp. 447-450, 1949.

[2] W. J. van Stockum, "IX.-The gravitational field of a distribution of particles rotating about an axis of symmetry," Proceedings of the Royal Society of Edinburgh, vol. 57, pp. 135-154, 1938.

[3] F. J. Tipler, "Rotating cylinders and the possibility of global causality violation," Physical Review D, vol. 9, no. 8, pp. 2203-2206, 1974.

[4] C. W. Misner and A. H. Taub, "A singularity-free empty universe," Soviet Physics JETP, vol. 28, p. 122, 1969.

[5] C. W. Misner, "The flatter regions of Newman, Unti, and Tamburino's generalized Schwarzschild space," Journal of Mathematical Physics, vol. 4, no. 7, pp. 924-937, 1963.

[6] A. H. Taub, "Empty space-times admitting a three parameter group of motions," Annals of Mathematics, vol. 53, no. 3, p. $472,1951$.

[7] E. Newman, L. Tamburino, and T. Unti, "Empty-space generalization of the Schwarzschild metric," Journal of Mathematical Physics, vol. 4, no. 7, pp. 915-923, 1963.

[8] R. P. Kerr, "Gravitational field of a spinning mass as an example of algebraically special metrics," Physical Review Letters, vol. 11, no. 5, pp. 237-238, 1963.

[9] B. Carter, "Global structure of the Kerr family of gravitational fields," Physics Review, vol. 174, no. 5, pp. 1559-1571, 1968. 
[10] A. Tomimatsu and H. Sato, "New series of exact solutions for gravitational fields of spinning masses," Progress of Theoretical Physics, vol. 50, no. 1, pp. 95-110, 1973.

[11] J. R. Gott, "Closed timelike curves produced by pairs of moving cosmic strings: exact solutions," Physical Review Letters, vol. 66, no. 9, pp. 1126-1129, 1991.

[12] J. D. E. Grant, "Cosmic strings and chronology protection," Physical Review D, vol. 47, no. 6, pp. 2388-2394, 1993.

[13] S. V. Krasnikov, "A singularity-free WEC-respecting time machine," Classical and Quantum Gravity, vol. 15, no. 4, pp. 997-1003, 1998.

[14] W. B. Bonnor, "The interactions between two classical spinning particles," Classical and Quantum Gravity, vol. 18, no. 7, pp. 1381-1388, 2001.

[15] W. B. Bonnor, "An exact, asymptotically flat, vacuum solution of Einstein s equations with closed timelike curves," Classical and Quantum Gravity, vol. 19, no. 23, pp. 5951-5957, 2002.

[16] W. B. Bonnor, "Closed Timelike Curves in Classical Relativity," International Journal of Modern Physics D, vol. 12, no. 9, pp. 1705-1708, 2003.

[17] W. B. Bonnor and B. R. Steadman, "The double-Kerr solution," Classical and Quantum Gravity, vol. 21, no. 11, pp. 2723-2732, 2004.

[18] P. Collas and D. Klein, "Letter: causality violating geodesics in Bonnor's rotating dust metric," General Relativity and Gravitation, vol. 36, no. 11, pp. 2549-2557, 2004.

[19] W. B. Bonnor and B. R. Steadman, "Exact solutions of the Einstein-Maxwell equations with closed timelike curves," General Relativity and Gravitation, vol. 37, no. 11, pp. 1833-1844, 2005.

[20] M. Gürses, A. Karasu, and Ö. Sarioğlu, "Gödel-type metrics in various dimensions," Class. Quantum Grav, vol. 22, no. 9, pp. 1527-1543, 2005.

[21] V. M. Rosa and P. S. Letelier, "Spinning strings, black holes and stable closed timelike geodesics," International Journal of Theoretical Physics, vol. 49, no. 2, pp. 316-323, 2010.

[22] J. Evslin and T. Qiu, "Closed timelike curves in the Galileon model," Journal of High Energy Physics, vol. 2011, p. 32, 2011.

[23] D. Sarma, M. Patgiri, and F. U. Ahmed, "Pure radiation metric with stable closed timelike curves," General Relativity and Gravitation, vol. 46, no. 1, p. 1633, 2014.

[24] F. Ahmed, B. B. Hazarika, and D. Sarma, "The anti-de Sitter spacetime as a time machine," The European Physical Journal Plus, vol. 131, no. 7, p. 230, 2016.

[25] F. Ahmed, "A stationary cylindrically symmetric spacetime which admits CTCs and its physical interpretation," Annals of Physics, vol. 386, pp. 25-33, 2017.

[26] F. Ahmed, "Cylindrically symmetric pure radiation space-time and closed timelike geodesics," Progress of Theoretical and Experimental Physics, vol. 2017, article 043E02, 2017.

[27] F. Ahmed, "A type D non-vacuum spacetime with causality violating curves, and its physical interpretation," Communications in Theoretical Physics, vol. 68, no. 6, p. 735, 2017.

[28] F. Ahmed, "Closed timelike curves in type II non-vacuum spacetime," Communications in Theoretical Physics, vol. 67, no. 2, p. 189, 2017.

[29] F. Ahmed, "Gravitational collapse of type N spacetime, the naked singularity, and causality violation," Progress of Theoretical and Experimental Physics, vol. 2017, article 083E03, 2017.

[30] F. Ahmed, "Type N Einstein space time machine spacetime," Annals of Physics, vol. 382, pp. 127-135, 2017.
[31] F. Ahmed, "A four-dimensional generalization of Misner space in curved spacetime," Journal of Physics Communications, vol. 2, no. 3, article 035036, 2018.

[32] F. Ahmed, "Axisymmetric pure radiation space-time with causality-violating geodesics," Theoretical and Mathematical Physics, vol. 195, no. 3, pp. 916-922, 2018.

[33] F. Ahmed, "A type $\mathrm{N}$ radiation field solution with $\backslash(\backslash$ Lambda $<0 \backslash)$ in a curved space-time and closed time-like curves," European Physical Journal C, vol. 78, no. 5, p. 385, 2018.

[34] F. Ahmed, "A family of type $\mathrm{N}$ space-time with a negative cosmological constant and causality violation," Progress of Theoretical and Experimental Physics, vol. 2019, article 013E03, 2019.

[35] F. Ahmed, "Axially Symmetric Type N Space-Time with a Naked Curvature Singularity and Closed Timelike Curves," Gravitation and Cosmology, vol. 26, no. 2, pp. 136-143, 2020.

[36] F. Ahmed, "Axially Symmetric Type N Space-Time with Causality Violating Curves and the von Zeipel Cylinder," Gravitation and Cosmology, vol. 26, no. 3, p. 265, 2020.

[37] A. Ori, "A class of time-machine solutions with a compact vacuum core," Physical Review Letters, vol. 95, no. 2, article 021101, 2005.

[38] M. S. Morris, K. S. Thorne, and U. Yurtsever, "Wormholes, time machines, and the weak energy condition," Physical Review Letters, vol. 61, no. 13, pp. 1446-1449, 1988.

[39] M. S. Morris and K. S. Thorne, "Wormholes in spacetime and their use for interstellar travel: a tool for teaching general relativity," American Journal of Physics, vol. 56, no. 5, pp. 395-412, 1988.

[40] M. Alcubierre, "The warp drive: hyper-fast travel within general relativity," Classical and Quantum Gravity, vol. 11, no. 5, pp. L73-L77, 1994.

[41] A. E. Everett, "Warp drive and causality," Physical Review D, vol. 53, no. 12, pp. 7365-7368, 1996.

[42] A. E. Everett and T. A. Roman, "Superluminal subway: the Krasnikov tube," Physical Review D, vol. 56, no. 4, pp. 21002108, 1997.

[43] F. Lobo and P. Crawford, "Weak Energy Condition Violation and Superluminal Travel," in Current Trends in Relativistic Astrophysics, L. Fernández-Jambrina and L. M. GonzálezRomero, Eds., vol. 617 of Lecture Notes in Physics, SpringerVerlag, Berlin, Germany, 2003.

[44] A. Ori, "Must time-machine construction violate the weak energy condition?," Physical Review Letters, vol. 71, no. 16, pp. 2517-2520, 1993.

[45] A. Ori and Y. Soen, "Causality violation and the weak energy condition,” Physical Review D, vol. 49, no. 8, pp. 3990-3997, 1994.

[46] Y. Soen and A. Ori, "Improved time-machine model," Physical Review D, vol. 54, no. 8, pp. 4858-4861, 1996.

[47] K. D. Olum, "Ori-Soen time machine," Physical Review D, vol. 61, no. 12, p. 124022, 2000.

[48] R. L. Mallett, "The gravitational field of a circulating light beam," Foundations of Physics, vol. 33, no. 9, pp. 1307-1314, 2003.

[49] K. D. Olum and A. E. Everett, "Can a circulating light beam produce a time machine," Foundations of Physics Letters, vol. 18, no. 4, pp. 379-385, 2005.

[50] A. Ori, "Formation of closed timelike curves in a composite vacuum/dust asymptotically flat spacetime," Physical Review $D$, vol. 76, no. 4, article 044002, 2007. 
[51] D. Sarma, F. Ahmed, and M. Patgiri, "Axially Symmetric, Asymptotically Flat Vacuum Metric with a Naked Singularity and Closed Timelike Curves," Advances in High Energy Physics, vol. 2016, 2546184 pages, 2016.

[52] F. Ahmed, "3587018Axially Symmetric Null Dust Space-Time, Naked Singularity, and Cosmic Time Machine," Advances in High Energy Physics, vol. 2017, 7 pages, 2017.

[53] F. Ahmed, "7943649Cylindrically Symmetric, Asymptotically Flat, Petrov Type D Spacetime with a Naked Curvature Singularity and Matter Collapse," Advances in High Energy Physics, vol. 2017, 6 pages, 2017.

[54] J. M. Maldacena, “The large \$N\$ limit of superconformal field theories and supergravity," Advances in Theoretical and Mathematical Physics, vol. 2, no. 2, pp. 231-252, 1998.

[55] C. W. Misner, Relativity Theory and Astrophysics I: Relativity and Cosmology, J. Ehlers, Ed., vol. 8 of Lectures in Applied Mathematics, American Mathematical Society, Providence, RI, USA, 1967.

[56] L.-X. Li, "Time machines constructed from anti-de Sitter space," Physical Review D, vol. 59, no. 8, article 084016, 1999.

[57] D. Levanony and A. Ori, "Extended time-travelling objects in Misner space," Physical Review D, vol. 83, no. 4, article 044043, 2011.

[58] M. Mars and J. M. M. Senovilla, "Axial symmetry and conformal Killing vectors," Classical and Quantum Gravity, vol. 10, no. 8, pp. 1633-1647, 1993.

[59] M. Mars and J. M. M. Senovilla, "On 'an infinite perfect fluid in cylindrically symmetric steady differential rotation'," Classical and Quantum Gravity, vol. 12, no. 8, pp. 2071-2076, 1995.

[60] J. C. N. de Araujo and A. Wang, "Rigidly rotating dust in general relativity," General Relativity and Gravitation, vol. 32, no. 10, pp. 1971-1980, 2000.

[61] H. Stephani, D. Kramer, M. MacCallum, C. Hoenselaers, and E. Herlt, Exact Solutions to Einstein's Field Equations, Cambridge University Press, Cambridge, 2005.

[62] I. D. Soares, "Inhomogeneous rotating universes with closed timelike geodesics of matter," Journal of Mathematical Physics, vol. 21, no. 3, pp. 521-525, 1980.

[63] F. A. E. Pirani, "On the Physical significance of the Riemann tensor," Acta Physica Polonica, vol. 15, p. 389, 1956.

[64] F. A. E. Pirani, "Invariant formulation of gravitational radiation theory," Physics Review, vol. 105, pp. 1089-1099, 1957.

[65] P. Szekeres, "The gravitational compass," Journal of Mathematical Physics, vol. 6, no. 9, pp. 1387-1391, 1965.

[66] J. Bičák and J. Podolský, "Gravitational waves in vacuum spacetimes with cosmological constant. II. Deviation of geodesics and interpretation of nontwisting type N solutions," Journal of Mathematical Physics, vol. 40, no. 9, pp. 4506-4517, 1999. 